\title{
Rosacea-associated Meibomian Gland Dysfunction - An Epidemiological Perspective
}

\author{
Eloy Viso, ${ }^{1}$ Alejandro Clemente Millán ${ }^{1}$ and María Teresa Rodríguez-Ares ${ }^{2}$
}

1. Staff Ophthalmologist, Servicio de Oftalmología, Complexo Hospitalario Universitario de Pontevedra, Pontevedra, Spain; 2. Professor of Ophthalmology, Servicio de Oftalmología, Hospital Clínico Universitario de Santiago de Compostela and Departamento de Oftalmología, Universidad de Santiago de Compostela, Santiago de Compostela, Spain

\begin{abstract}
The association between meibomian gland dysfunction (MGD) and rosacea is probably frequently underdiagnosed despite being one of the few associations of MGD that has been corroborated in population-based patients. Its diagnosis is not easy because the ocular signs and symptoms of this type of MGD are non specific and because the cutaneous signs may be minimal or absent; however, it is important because it may be associated with more severe manifestations such as keratitis, as several studies have shown, and a poorer prognosis. For this reason, clinical suspicion should be high especially in cases unresponsive to conservative measures and conventional treatment, and in those with inflammatory complications of the ocular surface.
\end{abstract}

\section{Keywords}

Association, dry eye disease, epidemiology, meibomian gland dysfunction, prevalence, rosacea

Disclosure: Eloy Viso, Alejandro Clemente Millán and María Teresa Rodríguez-Ares have no conflict of interest to declare. No funding was received for the publication of this article.

Received: 31 March 2014 Accepted: 7 May 2014 Citation: European Ophthalmic Review, 2014;8(1):13-6 DOI: 10.17925/EOR.2014.08.01.13 Correspondence: Eloy Viso, Oftalmología, Complexo Hospitalario Universitario de Pontevedra, 36003 Pontevedra, Spain. E: evo@mundo-r.com

Meibomian gland dysfunction (MGD) has recently been defined as a chronic, diffuse abnormality of the meibomian glands, commonly characterised by terminal duct obstruction and/or qualitative/ quantitative changes in the glandular secretion, which may result in alteration of the tear film, clinical apparent inflammation, ocular surface disease and symptoms of eye irritation. ${ }^{1}$ The association of this disorder with rosacea, a chronic cutaneous inflammatory disorder of both the vascular and tissue stroma characterised by episodes of exacerbation and remission of erythema, telangiectasia, papules or pustules affecting the convexities of the central face, is common in clinical practice and often results in a form of MGD that requires a different management approach to that recommended in MGD unrelated to rosacea. ${ }^{2,3}$ The link between these disorders remains elusive although some studies have suggested common pathophysiological pathways involving the upregulation of the innate immune system by microorganisms such as Demodex and Helicobacter pylori. ${ }^{2,4-6}$ The positive response of MGD and rosacea to azithromycin and doxycycline also suggests common pathogenetic mechanisms. ${ }^{7.8}$

Recent research has also identified other systemic factors that might be associated with MGD such as dyslipaemia or Sjögren syndrome ${ }^{9-11}$ but, in contrast to rosacea, most of them have not been submitted to epidemiological evaluation and are still being debated. In this paper we provide a summary of the most relevant studies on MGD and rosacea prevalence, and analyse the association between these disorders. such association has probably not been paid enough attention in the literature and in the clinic despite its frequency and clinical implications. The awareness of this diagnosis and its potential consequences is therefore warranted, since it remains to be commonly missed both in the dermatology and ophthalmology clinics. ${ }^{12}$

\section{Methods of Assessment}

The information on MGD pathophysiology provided by recent research has indicated the existence of analytic and descriptive features that are beginning to be investigated in diagnosis studies. Spectroscopic analyses have showed profiles that might become biomarkers of the disorder. ${ }^{13-16}$ Evaporimetry and inferometry and other techniques under investigation have also revealed characteristic patterns. ${ }^{17-19}$ However, these procedures are not applicable to epidemiological research because of their complexity and because they have not been standardised. That is why the biomicroscopic examination of the meibomian glands and meibomian secretions is still the preferred technique for the assessment of MGD in prevalence studies. ${ }^{20-25}$ The main advantage of this evaluation is its simplicity, since only a few signs of MGD, such as telangiectasias of the lid margin or MGD orifice plugging, are usually assessed, but it lacks objectivity as it depends entirely on the examiner's subjective evaluation. It also often leaves out one of the two categories of MGD described in the recent workshop. ${ }^{1}$ Additionally, the number of false positives may be higher than expected as normal individuals may present subtle changes on the lid margin indistinguishable from those of MGD patients. Other studies have used indirect indicators of MGD as the tear break-up time (TBUT) rather than direct observation, ${ }^{26}$ but this measurement may be abnormal in disorders other than MGD, such as allergic conjunctivitis or lid aperture disorders, and is not valid, although clinically useful, in this type of studies. Consequently, despite its 


\section{Table 1: Diagnostic Criteria for Rosacea (National Society Expert Committee, 2002)}

Presence of One or More of the Following Primary Features
Flushing (transient erythema)
Nontransient erythema
Papules and pustules
Telangiectasia
May Include One or More of the Following Secondary Features
Burning or stinging
Red plaques
Dry appearance
Oedema
Ocular manifestations
Peripheral location
Phymatous changes (most commonly rhinophyma)

limitations, a thorough examination of MGD at the slit lamp seems the most appropriate method of assessment. Uniform criteria, both in quantitative and qualitative terms, should be established, in this regard, in order to better delineate the prevalence of MGD in future investigations.

Recent studies have also yielded valuable information on rosacea pathophysiology, but unfortunately they have not provided biomarkers or the background for the development of specific diagnostic tests. The identification of rosacea cases relies therefore, as in MGD, on clinical signs alone. However, this diagnosis is not as controversial as that of MGD, especially since the publication of internationally accepted criteria for diagnosis and classification of rosacea in 2002 (see Table 1). ${ }^{27}$ This publication describes primary and secondary features as well as four rosacea subtypes and one variant. Primary features include cutaneous manifestations typical of rosacea, whereas secondary features include signs and symptoms that often appear with cutaneous signs, The presence of at least one primary feature is, according to these guidelines, the necessary condition to make a positive diagnosis. In the absence of more accurate diagnostic procedures, rosacea-associated MGD patients would have to meet both MGD and rosacea clinical criteria to be regarded as such, which may be a source of bias in prevalence studies as a group of patients with minimal or no cutaneous signs might be excluded from the analyses.

\section{Population-based Estimations}

Population-based approaches minimise selection bias and are thus the most appropriate design to investigate prevalence estimations. This design has yielded valuable data on the epidemiology of several ocular surface disorders, such as dry eye disease, of which MGD is a precipitating factor and major cause, ${ }^{28}$ but the information provided on MGD is limited because there are only a few studies on this disorder and because most of them fail to provide age and sex specific data. ${ }^{29}$ These studies reveal a striking variation in the prevalence of MGD probably because of the differences in the criteria used to diagnose the disease (see Table 2), but most of them also fail to provide analysis of associated factors. The highest prevalence rates, ranging from 51.8 to $69.3 \%$, are observed in Asian studies. ${ }^{21,22,24}$ The lower prevalence rates observed in white populations, ${ }^{20,25,26}$ which range from 3.5 to $30.5 \%$, might be explained by the more restricted criteria used to diagnose the disorder, although they may also be associated with other factors such as ethnicity. Multiracial studies would help clarify if the differences between Asian and western populations respond to methodological aspects or rather to genetic or environmental factors. Conflicting results have been reported regarding the association with sex and age. In a recent population-based investigation performed in Spain, an association with the male sex was detected, but this association was not significant when MGD unrelated to rosacea or asymptomatic patients were excluded from the analysis. ${ }^{25}$ This lack of association is consistent with the lack of association found in clinic-based investigations, since in this type of studies, participants are mostly symptomatic. An association between MGD and age was also found in the Spanish study. However, this association was not corroborated in a population-based survey performed in an Asian population, which might be explained by the likely higher prevalence of the hypersecretory category, more common in younger individuals, in this ethnic group. ${ }^{24}$

Population-based investigations have also provided confirmation of the association of MGD with rosacea, ${ }^{25}$ but not information on the prevalence of rosacea-associated MGD. These data must be estimated indirectly from studies on MGD and rosacea prevalence ${ }^{30}$ and from studies on MGD in rosacea patients (see Table 3). The prevalence of rosacea in clinic-based studies and retrospective databases ${ }^{31-33}$ report lower rates than expected by clinical practice. This may be explained in part by the lack of diagnostic criteria often observed in these investigations, which may lead to identify only the more severe cases. A female predominance is also observed in patients between 30 and 50 years in these studies, although this sex distribution is not observed in patients with ocular manifestation or in older individuals. Estimations from investigations specifically designed to determine the prevalence of rosacea, conducted by dermatologist, with diagnostic criteria consistent with the criteria recently published, provide similar age and sex data but higher prevalence rates.34-36 These studies are more reliable than clinic-based studies and databases, and their results, which range from 10 to $22 \%$, may be considered acceptable estimations of the prevalence of rosacea in the adult population.

Given the prevalence of MGD and rosacea, and MGD being a disorder that affects more than $80 \%$ of rosacea patients in most case control series, $^{37-40}$ rosacea might be an associated disorder in a substantial proportion of MGD patients. Additionally, although according to the recent criteria rosacea-associated MGD could only be diagnosed in the presence of simultaneous cutaneous manifestations, there are a number of patients with minimal cutaneous signs or even without them considered under this diagnosis who might increase this proportion. These patients usually remain without a definitive diagnosis for a long time, which is of great concern especially in the paediatric population because children present without cutaneous signs more commonly than other age groups. ${ }^{41,42}$ Rosacea should thus be considered a potential diagnosis in any child who has any combination of ocular manifestations such as blepharitis, keratitis or recurrent chalazia, and ocular surface-related symptoms. This type of MGD could also be a potential source of selection bias as it could further underestimate the prevalence of rosacea-associated MGD in studies performed in adult populations. However, well-conducted epidemiological research is needed to clarify the impact of this type of MGD in the paediatric as well as in the general population.

\section{Epidemiological Characteristics of Rosacea- associated Meibomian Gland Dysfunction}

Population-based research has shown that rosacea-associated MGD, unlike MGD unrelated to rosacea, is usually associated with symptoms that can seriously affect the quality of life. ${ }^{25,43,44}$ For this reason, the work-up of patients with ocular surface-related symptoms should include rosacea as a possible associated diagnosis, especially in patients with signs of MGD. 
Table 2: Prevalence of Meibomian Gland Dysfunction in Population-based Studies

\begin{tabular}{|c|c|c|c|c|c|c|}
\hline Study & Country & Number & Age (Years) & Prevalence & Diagnostic Criteria & Association with Rosacea \\
\hline \multirow[t]{2}{*}{ McCarty ${ }^{26}$ (1998) } & Australia & 926 & $40-97$ & $19.9 \%$ & TBUT $<10$ second & NA \\
\hline & & & & $8.6 \%$ & TBUT $<8$ second & NA \\
\hline Schein $^{20}(1997)$ & US & 2,482 & $>65$ & $3.5 \%$ & MG plugging/collarettes & NA \\
\hline $\operatorname{Lin}^{21}(2003)$ & Taiwan & 1,361 & $>65$ & $60.8 \%$ & MG plugging/telangiectasia & NA \\
\hline \multirow[t]{2}{*}{$\mathrm{Jie}^{22}(2009)$} & China & 1,957 & $40-84$ & $68 \%$ & Telangiectasia (symptomatic) & NA \\
\hline & & & & $69.3 \%$ & Telangiectasia (asymptomatic) & NA \\
\hline $\operatorname{Han}^{23}(2011)$ & Korea & 657 & $65-95$ & $51.8 \%$ & MG plugging & NA \\
\hline $\operatorname{Siak}^{24}(2012)$ & Singapore & 3,271 & $40-80$ & $56.3 \%$ & MG plugging/telangiectasia & NA \\
\hline $\operatorname{Viso}^{25}(2012)$ & Spain & 654 & $40-96$ & $30.5 \%$ & MGD signs & $\begin{array}{l}\text { Asymptomatic }\left(\mathrm{OR}_{\mathrm{a}}: 2.12\right) \\
\text { Symptomatic }\left(\mathrm{OR}_{\mathrm{a}}: 3.5\right)\end{array}$ \\
\hline
\end{tabular}

$\overline{M G D}=$ meibomian gland dysfunction; $N A$ = not available; $O R_{a}=$ age and sex adjusted odds ratio; $T B U T$ = tear break-up time.

Table 3: Prevalence of Rosacea in Selected Populations and in Databases

\begin{tabular}{|c|c|c|c|c|c|}
\hline Study & Country & Sample & Number & Prevalence & Ocular Manifestations \\
\hline $\operatorname{Berg}^{34}$ (1989) & Sweden & Working population & 809 & $10 \%$ & NA \\
\hline $\mathrm{Doe}^{33}(2001)$ & UK & Clinic-based & 3,383 & $1.8 \%$ & NA \\
\hline Bamford ${ }^{31}(2006)$ & US & Database & 1,450 & $2.1 \%$ & NA \\
\hline Romanowicz ${ }^{32}$ (2008) & US & Database & $9,151,174$ & $1.34 \%$ & NA \\
\hline Abram 35 (2010) & Estonia & Working population & 348 & $22 \%$ & NA \\
\hline Augustin ${ }^{36}$ (2011) & Germany & Working population & 90,880 & $2.3 \%$ & NA \\
\hline
\end{tabular}

$N A=$ not available.

Table 4: Prevalence of Ocular Signs in Rosacea Patients

\begin{tabular}{|c|c|c|c|}
\hline Akpek $^{37}$ (1997) & Quarterman ${ }^{38}$ (1997) & Ghanem $^{39}(2003)$ & Patiño-Rodríguez ${ }^{40}$ (2012) \\
\hline MGD (81 \%) & MGD (76 \%) & MGD (85.2 \%) & MGD (83.6 \%) \\
\hline Blepharitis (65 \%) & Ciliary injection (67 \%) & Hyperaemia (40.9 \%) & Hyperaemia (83.6 \%) \\
\hline Hyperaemia (45 \%) & Bulbar injection (58 \%) & Anterior blepharitis (44.3\%) & SPK (50.9 \%) \\
\hline Neovascularisation (16 \%) & Crusts/sleeves (58 \%) & Papillae (27.3\%) & Pannus (20\%) \\
\hline SPK (15 \%) & SPK (39 \%) & Chalazia (14.8\%) & Infiltrates (16.4 \%) \\
\hline Chalazia (10 \%) & Scales/collarettes (27 \%) & Neovascularisation (11.4 \%) & Papillae (10.9 \%) \\
\hline Scarring (9 \%) & Papillae (18 \%) & Follicles (10.2 \%) & Chalazia (7.3 \%) \\
\hline Episcleritis (8 \%) & Neovascularisation (18 \%) & Trichiasis/lash loss (10.2 \%) & Ciliary injection (7.3 \%) \\
\hline REE (5 \%) & Tear foaming (18 \%) & Pannus (10.2 \%) & Corneal thinning (7.3\%) \\
\hline Corneal ulcer (5 \%) & Trichiasis/lash loss (12 \%) & Infiltrates (8 \%) & Corneal ulcer (5.4 \%) \\
\hline Iritis (2 \%) & Chalazia (3 \%) & Corneal thinning (5.7 \%) & Trichiasis/lash loss (3.6 \%) \\
\hline Scleritis (0.7 \%) & Infiltrates (3 \%) & Episcleritis (4.5\%) & Neovascularisarion (3.6\%) \\
\hline Granuloma (0.7 \%) & & Corneal ulcer (3.5 \%) & \\
\hline \multirow[t]{2}{*}{ Phlyctenule (0.7\%) } & & Scarring (2.3 \%) & \\
\hline & & Scleritis (1.2 \%) & \\
\hline
\end{tabular}

$M G D=$ meibomian gland dysfunction; $R E E=$ recurrent epithelial erosions; $S P K=$ superficial punctuate keratitis.

However, although myriad symptoms exist in relation to this disorder, which may be out of proportion to the findings, such as dryness, sandy sensation, burning or sticky eyes, as in other ocular surface disorders, attempts to identify specific ones have failed. . $5,38,40$ Consequently, symptom-based approaches are not helpful in the differential diagnosis of these patients or in the investigation of the epidemiological characteristics of this disorder.

MGD in rosacea patients is also usually associated with inflammation of the lid margin and conjunctiva as several case-series studies have demonstrated (see Table 4). ${ }^{37-40}$ These inflammatory processes cause changes in the surface epithelium and reduced tear production. ${ }^{25}$ This explains why rosacea-associated MGD shows an association with abnormal TBUT and positive vital stains in contrast to other ocular surface disorders. Quarterman et al. ${ }^{38}$ found mean TBUT in patients with rosacea to be more than three times lower than in controls. In this study TBUT was also inversely associated with the severity of the ocular manifestations and directly with treatment with oral doxycycline. The reduction in tear production observed in rosacea-associated MGD also shows why the schirmer test, which is usually normal in MGD, is also affected down the line in these patients. ${ }^{45}$ Therefore, the tear film abnormalities that lead to the breakdown of the lachrymal functional unit homeostasis are the result of a mixed mechanism that involves both increased tear evaporation and decreased secretion.

Inflammation may also be found in other structures of the ocular surface and more rarely in the sclera and intraocularly. ${ }^{37,40}$ The cornea is probably the most characteristic of these locations and superficial punctuate epiteliopathy the most commonly encountered disorder. Recurrent epithelial erosions are found in some series and they are thought to be associated with the elevated metalloproteinase activity in the tear fluid of these patients. Keratitis may lead to neovascularisation, deep scarring and thinning if left untreated and is the most frequent sight-threatening 
disorder in rosacea patients. Severe dry eye is often an associated disorder of keratitis and is a contributing factor, together with trichiasis, conjunctival fibrosis and other long-term ocular surface changes that occur in rosacea, for the development of complications such as perforation. Bacterial colonisation is also common and may contribute to ocular complications as well. Other sight-threatening disorders, such as scleritis or uveitis may be seen, but they are fortunately uncommon.

There is no correlation between the severity of the ocular manifestations and the severity of the cutaneous disease. ${ }^{38,46,47}$ There are cases with mild ocular manifestations with severe cutaneous manifestations and cases with severe ocular manifestations with hardly any cutaneous sign. The latter represent a diagnostic challenge because patients with this condition are usually oblivious of their cutaneous disease, which can be so subtle that they may require a therapeutic trial with oral tetracycline or consultation with a dermatologist to confirm the diagnosis. Clinical suspicion of this entity is crucial to prevent irreversible damage of the ocular surface. Dermatological consultation may also be helpful in patients with concomitant severe cutaneous disease whose therapy might require a multidisciplinary approach. In each case, the visual prognosis depends on the severity of the ocular manifestations and on how early ocular rosacea is diagnosed and treated.

\section{Conclusions}

Although there is a paucity of population-based epidemiological data regarding the associations of MGD, recent research suggests that this disorder may be strongly associated with rosacea and that rosacea may imply a poorer prognosis of MGD. Therefore, a thorough examination of the facial skin of MGD patients, especially of those irresponsive to conventional measures, is warranted to make an early diagnosis and thus prevent the development of complications. Rosacea should also be considered in the differential diagnosis of inflammatory disorders of the ocular surface, such as keratitis, even in the absence of cutaneous signs. Further investigation with uniformly accepted diagnostic criteria is needed to better characterise the prevalence and associations of rosacea-associated MGD and its clinical implications.
1. Nelson JD, Shimazaki J, Benítez del Castillo JM, et al., The international workshop on meibomian gland dys a report of the definition and classification subcommittee Invest Ophthalmol Vis Sci, 2011;52:1930-7.

2. Alvarenga $\mathrm{LS}$, Mannis MJ, Ocular rosacea, Ocul Surf, 2005;3:41-58.

3. Vieira AC, Höfling-Lima AL, Mannis MJ, Ocular Rosacea - a review, Arq Bras Oftalmol, 2012;75:363-9.

4. Steinhoff M, Buddenkotte J, Aubert J, et al., Clinical, cellular and molecular aspects in the pathophysiology of rosacea, I Investing Dermatol Symp Proc, 2011;15:2-11.

5. Jarmuda S, O'Reilly N, Zaba R, et al., Potential role of Demodex mites and bacteria in the induction of rosacea, J Med Microbiol, 2012;61:1504-10.

6. Wladis EJ, Carlson JA, Wang MS, et al., Toll-like receptors and vascular markers in ocular rosacea, Ophthal Plast Reconstr Surg, 2013;29:290-3.

7. Foulks GN, Borchman D, Yappert M, et al., Topical azithromycin therapy for meibomian gland dysfunction: clinical response and lipid alterations, Cornea, 2010;29:781-8.

8. Foulks GN Borchman D, Yappert M, et al, Topica azithromycin and oral doxycycline therapy of meibomian gland dysfunction: a comparative clinical and spectroscopic pilot study, Cornea, 2013;32:44-53

9. Shimakazi J, Goto E, Ono M, et al., Meibomian gland dysfunction in patients with Sjögren's syndrome Ophthalmology, 1998;105:1485-8.

10. Sullivan DA, Sullivan BD, Evans JE, et al., Androgen deficiency, meibomian gland dysfunction and evaporative dry eye, Ann N Y Acad Sci, 2002;966:211-22.

11. Dao AH, Spindle JD, Harp BA, et al., Association of dyslipidemia in moderate to severe meibomian gland dysfunction, Am I Ophthalmol, 2010:150:371-6.

12. Vieira AC Mannis Mt Ocular rosacea: common and commonly missed, I Am Dermatol, and commonly miss:

13. Joffre C, Souchier M, Gregoire S, et al., Differences in meibomian fatty acid composition in patients with meibomian gland dysfunction and aqueous-deficient dry eye, Br J Ophthalmol, 2008;92:116-9.

14. Vieira $\mathrm{AC}, \mathrm{An} \mathrm{HJ}$, Ozcan S, et al., Glycomic analysis of tear and saliva in ocular rosacea patients: the search for a biomarker, Ocul Surf, 2012;10:184-92

15. Borchman D, Foulks GN, Yappert MC, et al., Human meibum lipid conformation and thermodynamic changes with meibomian gland dysfunction, Invest Ophthalmol Vis SCi 2011:52:3805-17.
16. Travo A, Paya C, Déléris G, et al., Potential of FTIR spectroscopy for analysis of tears for diagnosis purposes, Anal Bioanal Chem, 2014:406:2367-76.

17. Eom Y, Lee JS, Kang SY, et al., Correlations between quantitative measurements of tear film lipid layer thickness and meibomian gland loss in patients with obstructive meibomian gland dysfunction and normal controls, Am J Ophthalmol, 2013:155:1104-10.

18. Zeev MS, Miller DD, Latkany R, Diagnosis of dry eye disease and emerging technologies, Clin Ophthalmol, 2014;8:581-90.

19. Goto E, Endo K, Suzuki A, et al., Tear evaporation dynamics in normal subjects and subjects with obstructive meibomia gland dysfunction, Invest Ophthalmol Vis Sci, 2003;44:533-9.

20. Schein OD, Muñoz B, Tielsch JM, et al., Prevalence of dry eye among the elderly, Am J Ophthalmol, 1997;124:723-8.

21. Lin PY, Tsai SY, Cheng CY, et al., Prevalence of dry eye among an elderly Chinese population in Taiwan: The Shihpai Eye Study, Ophthalmology, 2003;110:1096-101.

22. Jie $Y, X u L$, , Wu YY, et al., Prevalence of dry eye among adult Chinese in the Beijing Eye Study, Eye, 2009;23:688-93.

23. Han SB, Hyon JY, Woo SJ, et al., Prevalence of dry eye disease in an elderly Korean population, Arch Ophthalmol 2011;129:633-8.

24. Siak JJ, Tong L, Wong WL, et al., Prevalence and risk factors of meibomian gland dysfunction: The Singapore Malay Study Cornea, 2012;31:1223-8

25. Viso E, Rodríguez-Ares MT, Abelenda D, et al., Prevalence of asymptomatic and symptomatic meibomian gland dysfunction in the general population of Spain, Invest Ophthalmol Vis Sci, 2012;53:2601-6.

26. McCarty CA, Bansal AK, Livingston PM, et al. The epidemiology of dry eye in Melbourne, Australia, Ophthalmology, 1998;105:1114-9.

27. Wilkin J, Dahl M, Detmar M, et al., Standard classification of rosacea: report of the national rosacea society expert committee on the classification and staging of rosacea I Am Dermatol, 2002:46:584-7.

28. Viso E, Gude F, Rodríguez-Ares MT, The association of meibomian gland dysfunction and other common ocular diseases with dry eye: a population-based study in Spain Cornea, 2011;30:1-6.

29. Schaumberg DA, Nichols JJ, Papas EB, et al., The internation workshop on meibomian gland dysfunction: report of the subcommittee on the epidemiology of, and associated risk factors for meibomian gland dysfunction, Invest Ophthalmo Vis Sci, 2011;52:1994-2005.

30. Tan J, Berg M, Rosacea: current state of epidemiology, Am Acad Dermatol, 2013:69:S27-\$35.
31. Bamford JT, Gessert CE, Renier CM, et al., Childhood stye and adult rosacea, J Am Acad Dermatol, 2006;55:951-5.

32. Romanowicz M, Stephenson JJ, Del Rosso JQ, et al., Healthcare utilization and costs of patients with rosacea in an insured population, J Drugs Dermetol 2008:7:41-9.

33. Doe PT, Asiedu A, Acheampong JW, et al., Skin diseases in Ghana and the UK, Int I Dermatol, 2001;40:323-6.

34. Berg M, Liden S, An epidemiological study of rosacea, Acta Derm Venereol, 1989;69:419-23.

35. Abram K, Silm H, Oona $\mathrm{M}$, Prevalence of rosacea in an Estonian working population using a standard classification Acta Derm Venereol, 2010;90:269-73.

36. Augustin M, Herberger K, Hintzen S, et al., Prevalence of skin lesions and need for treatment in a cohort of 90889 workers, Br J Dermatol, 2011;165:865-73.

37. Akpek EK, Merchant A, Pinar V, et al., Ocular rosacea. Patient characteristics and follow up, Ophthalmology, 1997;104:1863-7.

38. Quarterman MJ, Johnson DW, Abele DC, et al., Ocular rosacea signs, symptoms, and tear studies before and after treatment with doxycycline, Arch Dermatol, 1997:133:49-54

39. Ghanem VC, Mehra N, Wong S, et al., The prevalence of ocular signs in acne rosacea: comparing patients from ophthalmology and dermatology clinics, Cornea, 2003;22:230-3.

40. Patiño-Rodríguez BE, Rodríguez-García A, Díaz JC, et al., External ocular surface changes in ocular rosacea patients, Rev Mex oftalmol, 2012;86:86-96

41. Chamaillard M, Mortemousque B, Boralevi F, et al., Cutaneous and ocular signs of childhood rosacea, Arch Dermatol, 2008;144:167-71.

42. Cetinkaya A and Akova YA, Pediatric ocular acne rosacea: long-term treatment with systemic antibiotics, Am J Ophthalmol, 2006:142:816-21.

43. Shiffman RM, Walt JG, Jacobsen $\mathrm{G}$, et al., Utility assessment among patients with dry eye disease, Ophthalmology, 2003;110:1412-9.

44. Mertzanis P, Abetz L, Rajagopalan K, et al., The relative burden of dry eye in patients' lives: a comparison to a US normative sample, Invest Ophthalmol Vis Sci, 2005;46:46-50.

45. Zengin $\mathrm{N}$, Tol H, Günduz K, et al., Meibomian gland dysfunction and tear film abnormalities in rosacea, Cornea, 1995;14:144-6.

46. Oltz M, Check J, Rosacea and its ocular manifestations, Optometry, 2011;82:92-103.

47. Lazaridou E, Fotiadou C, Ziakas NG, et al., Clinical and laboratory study of ocular rosacea in northern Greece J Eur Acad Dermatol Venereol, 2011;25:1428-31. 